\title{
Synchronization of fluctuating delay-coupled chaotic networks
}

\author{
Manuel Jiménez-Martín, ${ }^{1}$ Javier Rodríguez-Laguna, ${ }^{1}$ Otti D’Huys, ${ }^{2}$ Javier de la Rubia, ${ }^{1}$ and Elka Korutcheva ${ }^{1,3}$ \\ ${ }^{1}$ Departamento de Física Fundamental, UNED, Spain \\ ${ }^{2}$ Department of Mathematics, Aston University, B4 7ET Birmingham, United Kingdom \\ ${ }^{3}$ G. Nadjakov Inst. Solid State Physics, Bulgarian Academy of Sciences, 1784, Sofia, Bulgaria
}

(Dated: May 1, 2017)

\begin{abstract}
We study the synchronization of chaotic units connected through time-delayed fluctuating interactions. Focusing on small-world networks of Bernoulli and Logistic units with a fixed chiral backbone, we compare the synchronization properties of static and fluctuating networks in the regime of large delays. We find that random network switching may enhance the stability of synchronized states. Synchronization appears to be maximally stable when fluctuations are much faster than the timedelay, whereas it disappears for very slow fluctuations. For fluctuation time scales of the order of the time-delay, we report a resynchronizing effect in finite-size networks. Moreover, we observe characteristic oscillations in all regimes, with a periodicity related to the time-delay, as the system approaches or drifts away from the synchronized state.
\end{abstract}

PACS numbers: 05.45.-a, 89.75.Hc

Keywords: chaos synchronization, time-delay, time varying networks

\section{INTRODUCTION}

Cooperative behavior of chaotic systems in interaction can lead to the emergence of partial and local synchronization [1. An interesting problem in this context is the stability of the synchronized state, which is ruled by the topology of the interaction [2, 3]. In most settings, the coupling terms carry a finite time-delay due to the finite velocity of transmission of information. Yet, even for infinitely large time-delay the units can achieve zero-lag synchronization [4]. The paradigmatic time-delayed coupled systems capable of chaos synchronization are semiconductor lasers [5], with interesting applications in secure communication [9, 10]. The phenomenon might have relevance as well in neuroscience [11, 12.

A better understanding of chaos synchronization can be gained by studying simple chaotic systems, such as Bernoulli maps under single [13] or multiple [14] timedelays, for which the conditions for a stable synchronized state can be obtained analytically. These studies generally perform a stability analysis of the synchronized state on a fixed interaction network. Additionally, a general formalism has been developed for ensembles of static random interaction networks [15].

Currently, there is an increasing interest in studying networks as time-varying entities [16]. In fact, network fluctuations are essential features of some systems such as, for instance, interacting neurons, where synaptic plasticity continuously changes the topology [17. It is interesting then to inquire how a fluctuating network affects synchronization stability [18. Recently, there has been a number of studies concerning synchronization on timevarying contact networks, where the topology changes due to the random motion of the agents, and the couplings are instantaneous. Most of them consider diffusive coupling of moving oscillators [19, 20, 22, but also chaotic units 21]. This problem has also been tackled for genetic oscillators moving on lattices 23,24 .
In this paper we study the effect of a fluctuating topology on the synchronization of chaotic units with nondiffusive, time-delayed couplings. There has been a recent work concerning the case of chaotic maps interacting on fluctuating topologies with short time-delays 25]. In our work, in contrast, we focus on the case of large time delays [3, 7], and explore synchronization stability on the full range of possible scalings between the time-delay and the network switching time scale.

We consider a system of coupled chaotic maps with a single coupling delay, $T_{d}$, where the coupling network fluctuates with a characteristic switching time $T_{n}$. These network fluctuations are random, and not adaptive, i.e., the network evolution is not linked to the state in any way. A general description of our system, as well as the chosen synchronization measures, are given in Section III. In order to understand the implications of a fluctuating topology, we first study the synchronization stability of static small-world networks in section III. Then, we thoroughly explore the case of time varying networks in section [V] The slow fluctuations regime, $T_{n} \gg T_{d}$, where the dynamics always have enough time to adapt to the current network, is described in section IV A. Section IV B presents the fast fluctuation regime, $T_{n} \ll T_{d}$, where we observe an enhancement of synchronization stability. This phenomenon has not previously been reported on weakly chaotic systems, and is in qualitative agreement with the fast switching approximation for diffusively coupled systems [26]. A synchronizing finite-size effect when $T_{n}$ is larger than $T_{d}$ is reported in section IVC. In all regimes we have observed the presence of characteristic oscillations on the synchronization level. These are described and studied on section IVD. Finally, the last section is devoted to the conclusions and further work. 


\section{SYNCHRONIZATION OF DELAYED CHAOTIC NETWORKS}

Let us consider $N$ classical units, characterized by a single degree of freedom $u_{i}(t), i \in\{1, \cdots, N\}$ and time $t \in \mathbb{N}$, whose evolution is given by

$$
u_{i}(t+1)=(1-\epsilon) f\left(u_{i}(t)\right)+\epsilon \sum_{j} G_{i j}(t) f\left(u_{j}\left(t-T_{d}\right)\right) .
$$

Here, $\epsilon \in[0,1]$ is a real parameter which measures the strength of the interaction, $T_{d}$, is the coupling delay and $f:[0,1] \mapsto[0,1]$ is a chaotic map. We consider the Bernoulli map, modeled as

$$
f(x)=a x \bmod 1,
$$

with $a \in \mathbb{R}^{+}$, and the Logistic map, given by

$$
f(x)=r x(1-x)
$$

with $r \in \mathbb{R}^{+}$. The coupling topology is encoded in the network adjacency matrix $G$. The only requirement at this point for the coupling weights is to satisfy a stochasticity condition, such that the existence of a synchronized solution $u_{i}(t)=s(t)$ is guaranteed,

$$
\sum_{j} G_{i j}(t)=1
$$

for all $i$ and all times $t$. Nonetheless, this does not inform us about the stability of such synchronized state.

As a measure of the (zero-lag) synchronization in the network, we have chosen the logarithm of the spatial deviation over the network nodes. Let us explain in detail the meaning of this observable. Consider the spatial average of the unit states for a given time as

$$
\mu(t) \equiv \frac{1}{N} \sum_{i=1}^{N} u_{i}(t)
$$

and the corresponding spatial standard deviation as

$$
\sigma \equiv \sqrt{\frac{1}{N} \sum_{i=1}^{N}\left(u_{i}-\mu\right)^{2}}
$$

Then, the synchronization level is defined as

$$
\mathcal{S} \equiv-\ln (\sigma)
$$

A perfectly synchronized state would have $\mathcal{S} \rightarrow+\infty$, but in practice this value is bounded by the machine precision. In our calculations, using double precision floating point numbers, the maximal synchronization level corresponds approximately to $\mathcal{S} \sim 35$, which implies a deviation of order $\sigma \sim \exp [-35] \sim 10^{-15}$. On the other extreme, in a desynchronized state each unit behaves independently and $\mathcal{S}=O(1)$. The minimal computed value of $\mathcal{S}$ is close to 1.95 , which is close to the mean deviation for a uniform distribution on $[0,1]$ : $-\ln \sigma=\ln (12) / 2 \approx 1.24$.

We initialize the network close to the synchronized state: all units evolve in unison for $T_{d}$ time steps and, at $t=0$, we apply a random point-like perturbation, $\boldsymbol{\xi}=A \cdot \mathbf{r}$, where $A=10^{-10}$ and $\mathbf{r}$ is a vector of random numbers drawn uniformly from $[0,1]$. Thus, the synchronization level at $t=0$ is always around 24, instead of the machine precision value of 35 . We will denote by $\langle\cdot\rangle$ the realization average over such initial conditions. Our most relevant observable, therefore, will be $\langle\mathcal{S}(t)\rangle$.

We define a synchronization Lyapunov exponent (SLE) as the average linear rate at which the synchronization level increases or decreases with time:

$$
\langle\mathcal{S}(t)\rangle \sim \mathcal{S}_{0}-\lambda t
$$

By this definition, $\lambda$ is equivalent to the maximal Lyapunov exponent transverse to the synchronization manifold, given by the master stability function [2, which approximates the evolution of a perturbation from the synchronized state $\sigma \sim\left(u_{i}-\mu\right) \propto e^{\lambda t}$. A negative SLE value implies stable synchronization, as perturbations decay exponentially towards the synchronized state, while a positive SLE makes the synchronized state unstable. We will refer to networks with negative (positive) SLE as synchronizing (non-synchronizing).

The stability of the synchronized state is related to the second largest eigenvalue of the adjacency matrix $G$. Let $\left\{\gamma_{i}\right\}_{i=1}^{N}$ be the eigenvalues of $G$ sorted in descending order of their modulus, $\left|\gamma_{1}\right| \geq\left|\gamma_{2}\right| \geq \cdots \geq\left|\gamma_{N}\right|$. Gerschgorin circle theorem [27] can be applied, showing that $\left|\gamma_{i}\right| \leq 1$, and the unit row sum guarantees that $\left|\gamma_{1}\right|=1$, with eigenvector $v_{1}=[1, \ldots, 1]$. Hence, a perturbation along this mode preserves synchronization as it affects every unit equally. The evolution of a perturbation away from the synchronization manifold will then evolve according to the mode with second largest eigenvalue [2].

In networks coupled with a large interaction delay, synchronization is only possible in the weak chaos regime. Bernoulli maps are weakly chaotic if $a(1-\epsilon)<1$ holds 7]. In this case the SLE can be approximated by the following expression [28].

$$
\lambda \approx \frac{1}{T_{d}} \ln \left|\frac{a \epsilon \gamma_{2}}{1-a(1-\epsilon)}\right|
$$

This expression is derived from the linear stability analysis of the perturbation mode associated with the second largest eigenvalue. Such a procedure is undertaken in the appendix B, and Eq. (9) can be derived from Eq. (B3). From the stability condition, $\lambda<0$, we get a time-delay 
(a)

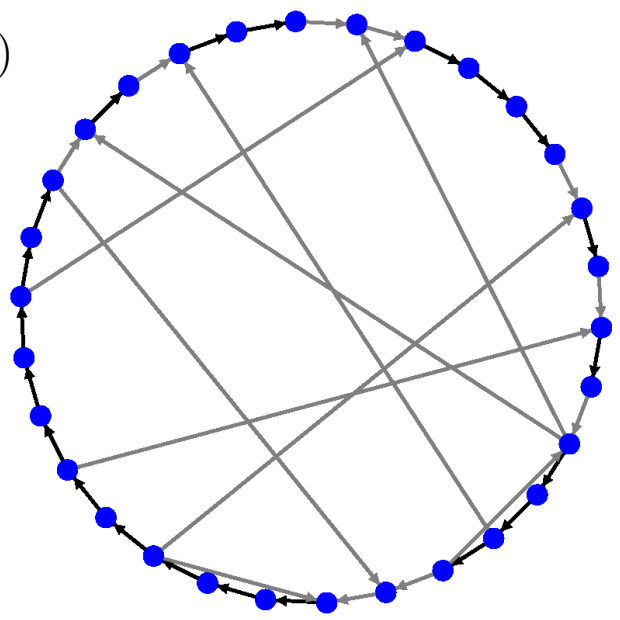

(b)

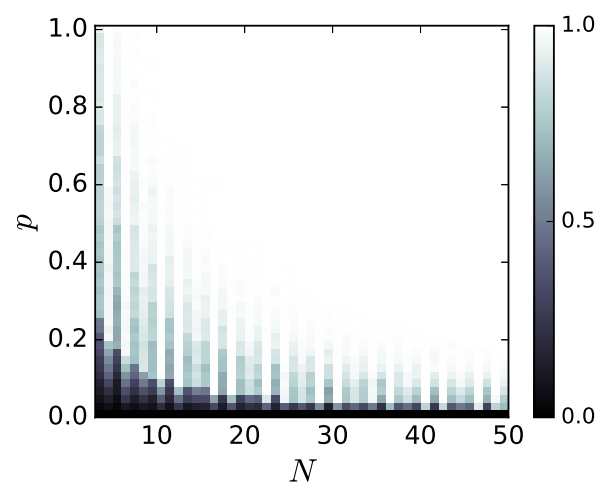

Figure 1: (a) A $N=30$ network with a clockwise rotating backbone and $p=0.3$, so the number of shortcuts is $N_{s}=9$. The strength of each link is denoted by its color: black is 1 and gray is $1 / 2$. Notice that the sum of input links on any node is always 1, as imposed in Eq. (4). (b) Average fraction of the networks having $G C D=1$ in the $N \times p$ space. For high enough number of units and shortcuts the probability of $G C D>1$ is negligible.

independent stability constraint for the coupling:

$$
\epsilon>\frac{a-1}{a \Delta}
$$

where $\Delta=1-\left|\gamma_{2}\right|$ is the spectral gap or eigengap.

For other chaotic maps, the stretch factor of the map $\left|f^{\prime}(x)\right|$ is not constant, and the SLE is not analytically accessible. However, it has been shown that fluctuations in the term $\left|f^{\prime}(x)\right|$ along the chaotic trajectory result in a larger spectrum of Lyapunov exponents and thus in a smaller parameter region that sustains stable synchronization 29]. We will study synchronization stability on fluctuating networks of coupled Bernoulli maps in order to be able to compare with the analytical results for static networks. We will also study networks of Logistic maps in order to assess the generality of our results.

\section{SYNCHRONIZATION OF SMALL-WORLD STATIC NETWORKS}

We have studied the stability of the synchronization manifold on statistical ensembles of small world networks, which constitute a standard benchmark for network synchronization [18, 30 32. Here, we consider a family of Newman-Watts networks [33, similar to the standard small-world networks 34 but keeping the outside ring fixed, so that it is guaranteed that the network is always connected. We will refer generically to these networks as small world (SW).

To construct our networks, we consider a chiral 1D ring of $N$ sites, where the only non-zero entries are $G_{i, i+1}$ for $i=1, \ldots N-1$, and $G_{N, 1}$. Then, we add to the network $\langle p N\rangle$ shortcuts, with $p \in[0,1]$. That is, for every node we establish a directed link to another randomly chosen node with probability $p$. An example of such a SW network, with $N=30$ and $p=0.3$ is shown in Fig. 1 (a). The resulting adjacency matrix is then row-normalized according to Eq. (4).

A well known result in delayed networks states that
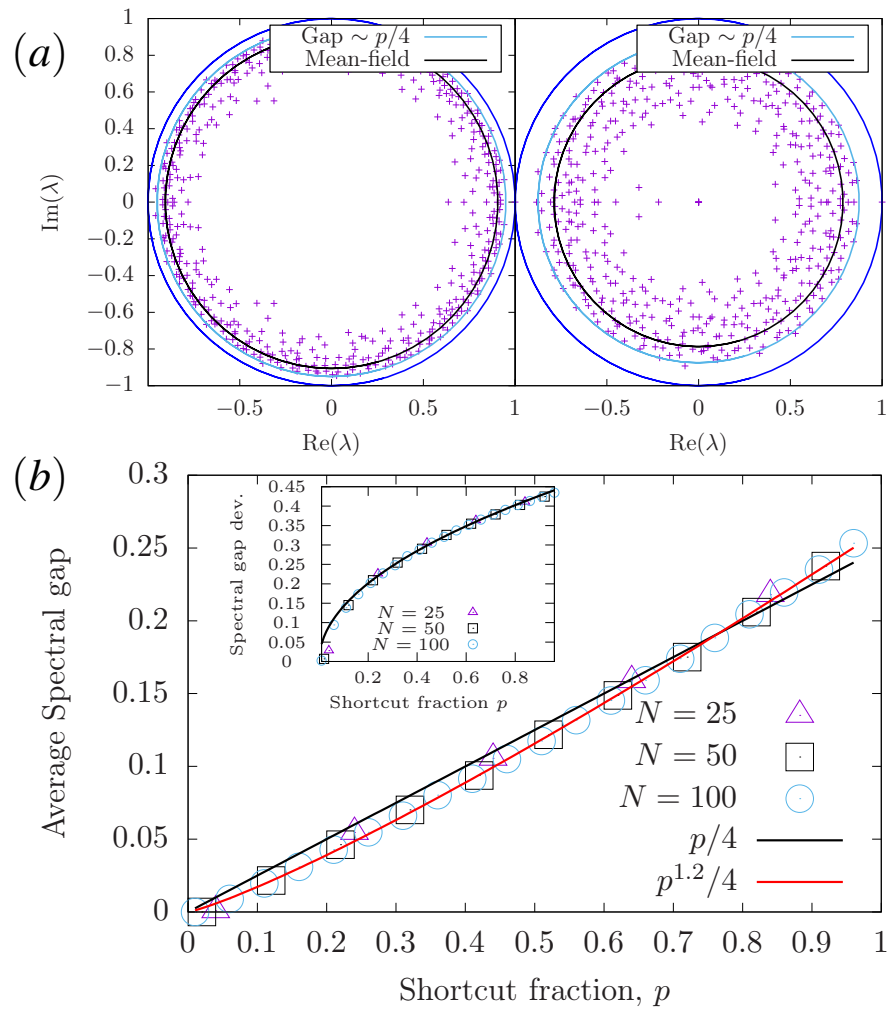

Figure 2: (a) Spectrum of the adjacency matrix of two SW networks, using $N=500$, and $p=1 / 5$ (left) and $1 / 2$ (right). Most of the eigenvalues are contained within a ring whose outer radius scales as $p / 4$. (b) spectral gap for different values of $N$ as a function of $p$ of our SW networks, which grows approximately as $p / 4$. A more accurate fit, with exponent 1.2 is also shown. Inset: Standard deviation of the eigengap, scaling as the square root of $p$. 
the number of possible stable synchronized subnetworks is equal to the Greatest Common Divisor (GCD) of the loop lengths of the network [13. Hence, complete synchronization is only possible if the GCD of the lengths of all cycles in the network is unity. This is almost always the case for large enough networks with a finite number of shortcuts, as can be seen in Fig. 1 (b). When the GCD condition is met, the stability of the synchronization manifold is still determined by the eigengap, as stated in Eq. 10 for the specific case of Bernoulli maps.

The adjacency matrices $G$ of directed networks are not hermitian, and their spectrum need not be real. Let us discuss the statistical properties of their spectra, in similarity to the studies of [32, 35]. Fig. 2 (a) shows the eigenvalues $\left\{\gamma_{i}\right\}$ on the complex plane for two SW networks, using $N=500$ and $p=1 / 5$ (left) and $1 / 2$ (right). Notice that, following Gerschgorin theorem, they are always contained within the unit circle. Except for the $\gamma_{1}=1$ eigenvalue, which is a consequence of the rowsum condition, the phases of the eigenvalues seem to be homogeneously distributed. Moreover, they seem to be contained within a ring, whose radius we would like to characterize.

An interesting approach to estimate the properties of the spectrum of random matrices describing SW networks was developed in [32, using a mean-field approach: write down the average matrix, whose entries are given by the average of the matrix entries. Due to translation invariance, the resulting matrix is a circulant matrix, whose spectrum can be found analytically. We have followed this approach in order to find the mean field spectrum of our SW networks. In the appendix A we show that the spectrum of this average matrix lies in the vicinity of a circumference of radius

$$
\left|\gamma_{m}^{\mathrm{MF}}\right| \approx \frac{1-\mathrm{e}^{-p}}{p}
$$

where $p$ is the shortcut probability. This expression is plotted in Fig. 2 (a) as mean-field line. However, the mean-field theory does not describe accurately the ring structure of the spectrum. We have found numerically that the outer circumference has an approximate radius of $(1-p / 4)$, independent of $N$. Thus, our estimate for the gap is

$$
\Delta \approx p / 4
$$

Fig. 2(b) shows the numerical evidence for expression (12), plotting the average spectral gap as a function of $p$ for different system sizes $N$. Interestingly, the inset shows that the standard deviation of the spectral gap among samples only grows like the square root of $p, \sigma_{\Delta} \sim p^{1 / 2} / 2$.

Once the spectral properties of our networks have been elucidated we can proceed to study their dynamics. We have simulated the dynamical system Eq. (1) and obtained numerically the synchronization Lyapunov exponent (SLE) for $10^{5}$ different SW networks with $N=40$,

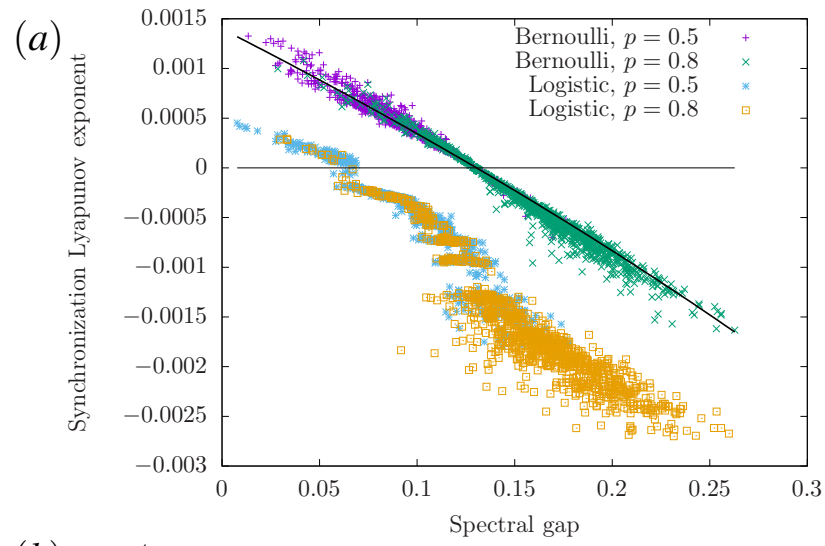

(b)

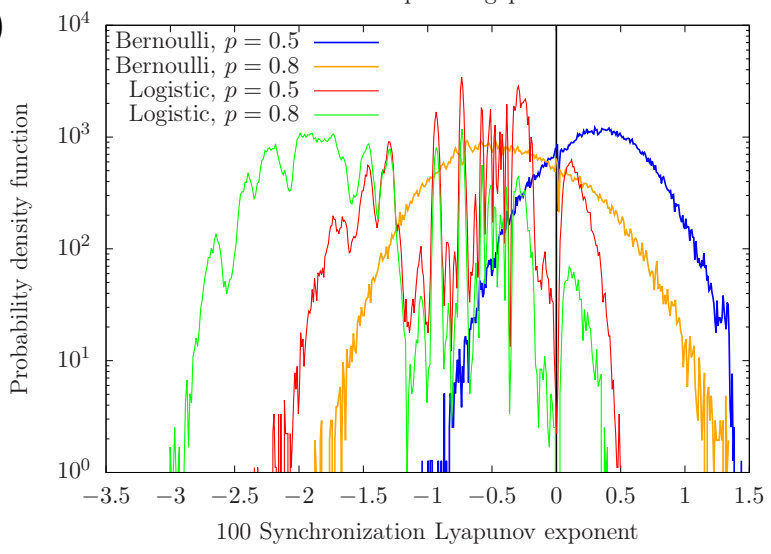

Figure 3: Measurement of the synchronization Lyapunov exponent (SLE) along with the spectral gap for $10^{5}$ samples of two SW network ensembles with $N=40$ sites, $\epsilon=0.7$, and $p=0.5$ and $p=0.8$, for two different dynamical systems: Bernoulli and Logistic, with parameters $a=1.1$ and $r=3.577$, respectively. (a) Relation between the SLE and the spectral gap. The black line corresponds to the theoretical expression Eq. 9 for coupled Bernoulli maps with long delays, and captures correctly the measured SLE, specially at values close to zero. For the Logistic maps, the correlation is still strong, but much more involved. (b) SLE histogram for the same four cases, in log-scale. Notice that, for Bernoulli we obtain an approximately Gaussian behavior, with non-zero skewness. For the Logistic case, the SLE are distributed in a much more complicated way. The vertical black bar marks the zero SLE.

$T_{d}=100, \epsilon=0.7$, two values of $p=0.5$ and 0.8 , and two maps: Bernoulli, Eq. (2), and Logistic, Eq. (3). We have chosen the numerical values of the parameters such that $a=1.1$ and $r=3.577$ so both maps have comparable Lyapunov exponents when considered in isolation [41.

In Fig. 3 (a) we plot the values of the SLE against the spectral gap of each network. In the Bernoulli case we find a tight relationship which follows approximately the theoretical expression for long delays Eq. (9). The Logistic case is more convoluted, but the negative correlation between both magnitudes is still clear. We can see that Logistic systems synchronize better than Bernoulli ones as they show a higher number of negative SLE values. Generally, networks with a larger spectral gap syn- 
(a)

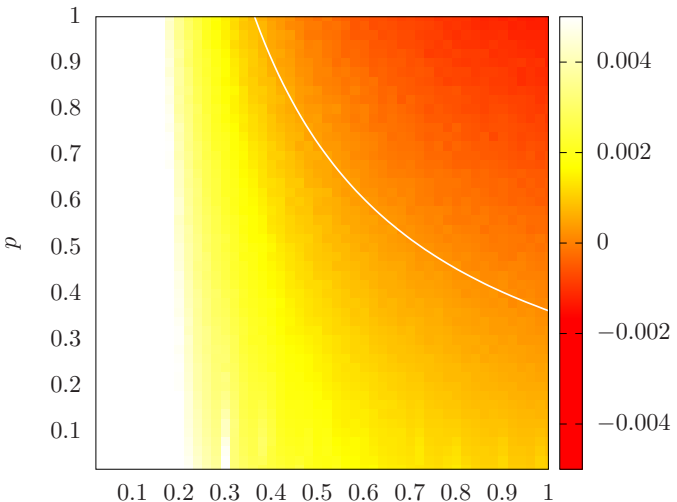

$\begin{array}{llllllllll}0.1 & 0.2 & 0.3 & 0.4 & 0.5 & 0.6 & 0.7 & 0.8 & 0.9 & 1\end{array}$

(b)

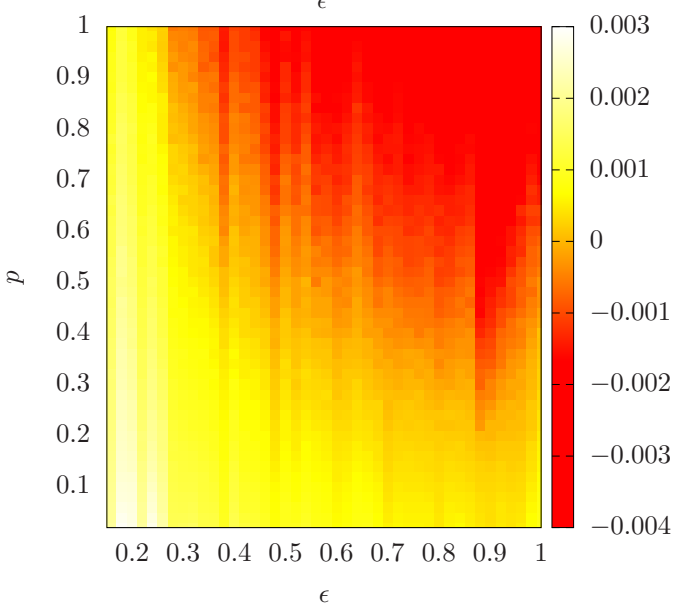

Figure 4: Average SLE for our SW networks as a function of both $\epsilon$ and $p$, using 100 samples for each point. (a) Bernoulli map, with $a=1.1$. (b) Logistic map with $r=3.577$. The white line delimits the theoretical synchronization region, Eq. 10 for a static network of Bernoulli maps and eigengap $\Delta=$ $p / 4$.

chronize better. Also, there is a higher probability of synchronization for higher $p$ values, since these networks present a larger eigengap.

Fig. 3 (b) shows the histogram of the SLE for Bernoulli and Logistic systems, using always $N=40, T_{d}=100, \epsilon=$ 0.7 and two values of $p=0.5$ and 0.8 . The black vertical bar marks the zero value: points on its left correspond to networks in which the synchronized state is stable. The histograms for the Bernoulli case have a nearly Gaussian shape, but with finite skewness and kurtosis [36. The histograms are much more complex for the Logistic case.

We have also performed a thorough exploration of the $(\epsilon, p)$-parameter space. In Fig. 4, we plot the average value of the SLE after 100 samples for each point, for the Bernoulli (top) and for the Logistic (bottom) maps. Negative SLE allows for stable synchronization, while positive values drive the system away from the synchronized state. The white line represents the theoretical synchronization line for networks of Bernoulli maps, Eq. (10), for an eigenvalue gap of $\Delta=p / 4$, and follows the zero average SLE accurately.

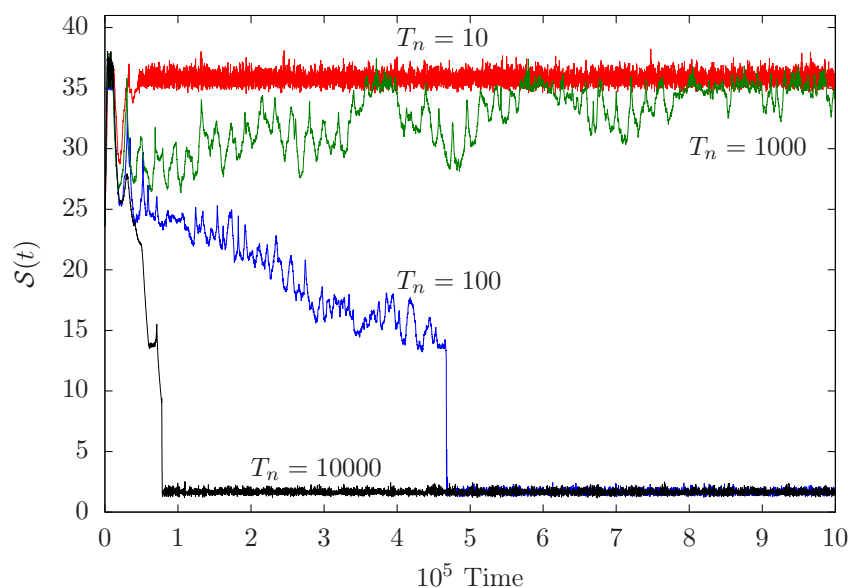

Figure 5: Synchronization level histories for a fluctuating SW network of Bernoulli maps with different fluctuation times. Parameter values are $a=1.1, N=40, p=0.5, T_{d}=100$ and $\epsilon=0.7$.

\section{FLUCTUATING NETWORKS}

Let us allow our networks to fluctuate, making $G$ time-dependent with a network switching period $T_{n}$ : the coupling topology will switch from the current network, $G_{\text {curr }}$, to a newly sampled $G_{\text {next }}$ every $T_{n}$ time steps. We sample from the ensemble of all SW graphs with fixed $N$ and $p$ as defined in section III.

As a first attempt, we see in Fig. 5 a few time traces of the synchronization level, $\mathcal{S}$, for fluctuating SW networks of Bernoulli maps with different fluctuation times: $T_{n}=10,10^{2}, 10^{3}$ and $10^{4}$. For this parameters choice the average SLE is positive albeit small: $\lambda=0.00022$. Unless otherwise stated, we will always choose $T_{d}=100$ for the time-delay. For $T_{n}=10^{4}$, the synchronization level decays to its minimal value fast. For $T_{n}=1000$, we observe strong fluctuations in the state deviation, but an ultimate synchronization. For $T_{n}=100$, when the fluctuation time-scale coincides with the delay, the system desynchronizes, although more slowly. Finally, for $T_{n}=10$, the system synchronizes fully quite fast.

In order to better understand the phenomenology, let us average the synchronization level for a large number of realizations, $N_{s}=1000$. Fig. 6 shows the results for three different systems of coupled Bernoulli maps. Fig. 6 (a) shows the average synchronization for the same system as in Fig. 5 in order to assess whether those results are generic. We see that for very short fluctuation time $T_{n}=1$ or 10 , the average synchronization level remains close to its maximum value. Hence the system synchronizes completely for all realizations independently of the sign of the SLE of the instantaneous networks. For $T_{n}=50$ the synchronization level decays very slowly with time, and it decays much faster for $T_{n}=100$. As we increase the fluctuation time, for $T_{n}=500$, the synchronization decay is again a bit slower, and for $T_{n}=1000$ or 2000 we can see that the system does not seem to 


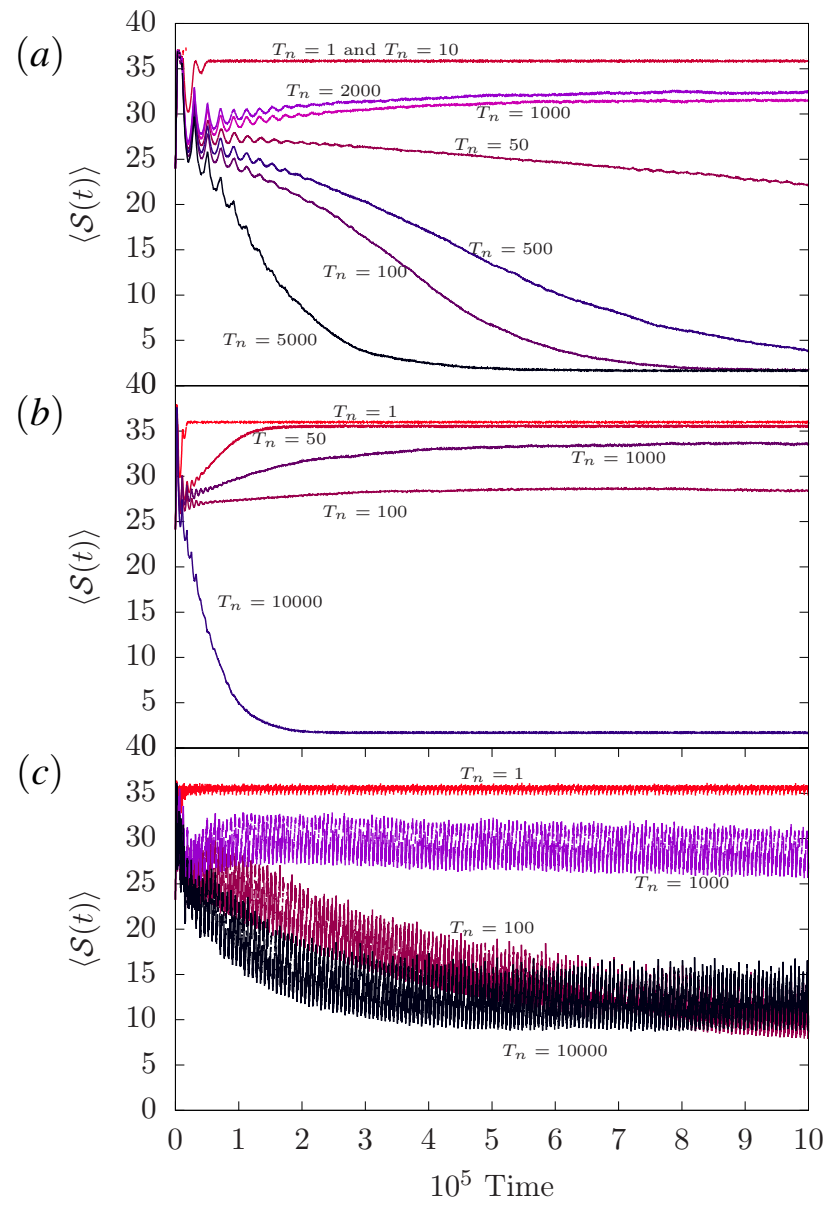

Figure 6: Realization average of the synchronization level, $\langle\mathcal{S}(t)\rangle$, Eq. (7), over $N_{s}=1000$ samples, as a function of time for different systems. (a) Bernoulli system, $a=1.1, N=40$, $p=0.5$ and $\epsilon=0.7$ (on average, non-synchronizing), for different values of $T_{n}$. (b) Bernoulli system with $a=1.1, N=$ 40 sites, $p=0.8$ and $\epsilon=0.47$ (in average, synchronizing), for different values of $T_{n}$. (c) Logistic system, $r=3.577$, $N=40, p=0.5$ and $\epsilon=0.4$ (in average, non-synchronizing). In all cases, $T_{d}=100$, and we only show time steps which are multiples of 100 .

desynchronize, but stays at a lower level of synchronization. For even slower fluctuations, $T_{n}=5000$, the system desynchronizes again quite fast. We also remark the presence of oscillatory behavior for the synchronization level at short times after the initial perturbation.

Fig. 6] (b) shows the average synchronization level for a fluctuating SW network of Bernoulli maps at parameter values for which the average SLE is negative. The main difference we can observe is that for $T_{n}=100$ the system does not desynchronize, even though the asymptotic synchronization level is lower than for $T_{n}=50$ and $T_{n}=1000$. However, for very slow fluctuation time $T_{n}=10000$ the system still desynchronizes. The short time oscillations that we observed in the previous case are still present, but attenuated.

In Fig. 6 (c) we see the average synchronization level for a fluctuating network of logistic elements at a parameter choice laying a positive average SLE. The system synchronizes for fast network fluctuations $T_{n}=10$, and for intermediate network times $T_{n}=1000$, but not for $T_{n} \sim T_{d}$. Again, in the slow fluctuations regime, the synchronized state is unstable. Finally, we report much stronger oscillations for this case than for the Bernoulli system.

The analysis of figure 6 illustrates the phenomenology of our system in its different regimes. Generally, we observe desynchronization for slow fluctuations and synchronization enhancement in the fast switching regime. The case where $T_{n} \sim T_{d}$ is more complicated, showing a recovery of the synchronization level when $T_{n}$ is larger than $T_{d}$. We argue that this is a finite-size effect, that is no longer present when studying larger networks. Finally, we observe characteristic oscillations which decay after the initial perturbation. We analyze separately each of these cases in the subsequent sections.

\section{A. Slow fluctuations}

We have found out that, with all other parameters fixed, there is always a critical value $\epsilon^{*}$ such that, if $\epsilon>\epsilon^{*}$ the system stays synchronized almost surely. We

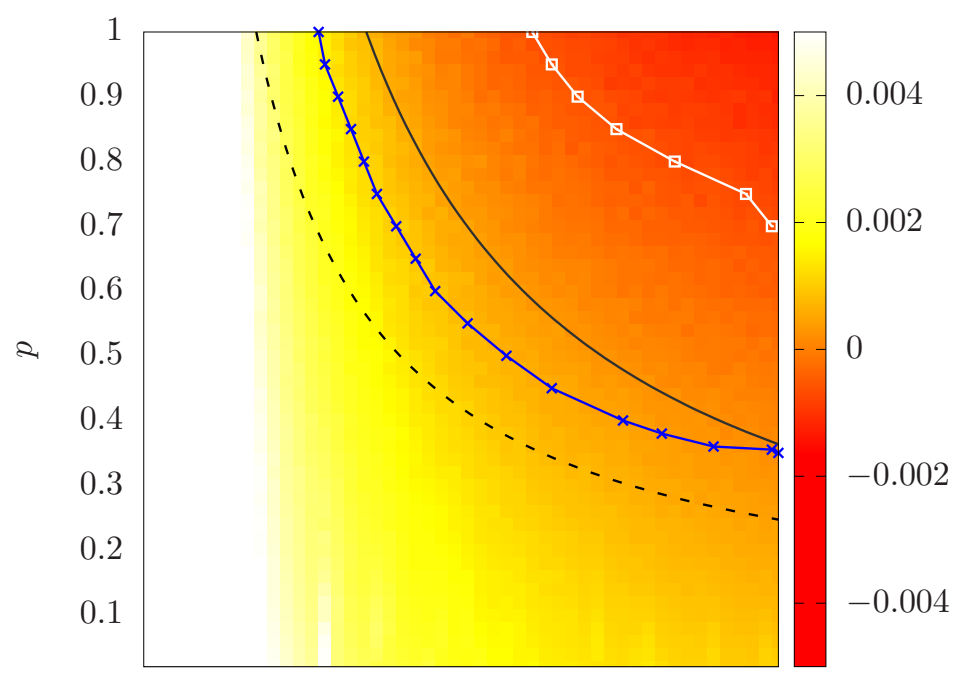

$\begin{array}{llllllllll}0.1 & 0.2 & 0.3 & 0.4 & 0.5 & 0.6 & 0.7 & 0.8 & 0.9 & 1\end{array}$

Figure 7: Synchronization regions in the parameter space for fluctuating networks of Bernoulli maps in different regimes $\left(a=1.1, T_{d}=100\right)$. The blue x's line denotes the $\epsilon^{*}$ values as a function of $p$ for fast fluctuations, $T_{n}=10$. The white, open-squares line denotes the $\epsilon^{*}$ value for very slow fluctuations, $T_{n}=10^{4}$. The dashed line denotes the meanfield approximation given by Eq. 11). For comparison with the static case, we have kept the background colormap corresponding to the static SLE from Fig. 4 (a), and the black continous line is the theoretical synchronization line from Eq. 10 
use the expression almost surely meaning that out of the $N_{s}=1000$ samples launched, all of them stayed synchronized up to time $10^{6}$. Figure 7 shows the critical $\epsilon^{*}$ curves as a function of $p$ for a system of Bernoulli maps for different network fluctuation times, $T_{n}$. The white, opensquares line in Fig. 7 shows the $\epsilon^{*}$ line for $T_{n}=10^{4}$. This corresponds to the slow fluctuations regime, $T_{n} \gg T_{d}$. In this regime the synchronization region shrinks to higher $\epsilon^{*}$ values, something that we anticipated from the slow fluctuations trajectories in Fig. 6. In this regime the coupling topology seen by the system is static during long periods. Then, one might expect the synchronization region to be identical to that corresponding to the static case, delimited by the white continuous line. However, the system does not synchronize even in regions where the average SLE is negative. The reason is that, for Bernoulli systems, desynchronization is irreversible in practice. When a non-synchronizing network is sampled the system will start to desynchronize. Once the system has evolved sufficiently far away from the synchronized state, the probability that a subsequently sampled synchronizing network takes the system back to synchronization is negligible. Thus, we may regard the long term dynamics as desynchronized whenever the probability of reaching a non-synchronizing network is finite.

\section{B. Fast fluctuations}

The blue x's line in Fig. 7 delimits the synchronization region for $T_{n}=10$, illustrating the fast fluctuation regime $T_{n} \ll T_{d}$. Interestingly, the critical line is below the average synchronization line for static networks, meaning that synchronization is stable for fast enough fluctuations even when the average SLE of the individual sampled networks is positive. This enhancement of the synchronization stability for rapidly fluctuating networks has been reported for a variety of systems with diffusive coupling [19 21]. Moreover, the fast switching approximation states that when the time-scale of the fluctuations is much larger than the typical time-scale of the oscillator dynamics, the synchronization properties are well described by a mean-field network 26]. That is not the case in the framework of time-delayed weakly chaotic systems: the dashed line in Fig. 7 delimits the synchronization region expected from the mean-field spectrum in Eq. (11). As we can see, it differs from the blue curve for fast network fluctuations, hence in our setting the meanfield curve does not provide a good approximation for the synchronization region of the fast switching regime.

In order to explain this synchronization enhancement by fast network fluctuations we might conjecture that networks with large eigengap pull the system towards the synchronization manifold, while low gap ones push it away. However, we have found that sufficiently fast fluctuations are able to synchronize the network even when every individually sampled network have a positive SLE. In Fig. 8 we consider the average synchronization level of
SW networks with $\epsilon<\epsilon^{*}$. Specifically, we reject all SW networks whose with eigengap $\Delta>0.1$, which gives a lower bound of at least $\epsilon^{*}>0.909$ for the individual networks. Yet, for fast network switching the system almost surely synchronizes.

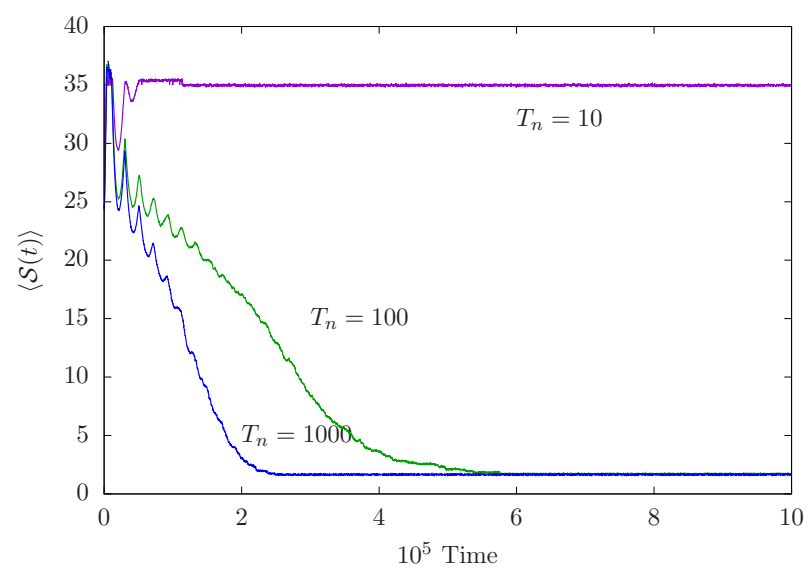

Figure 8: Average synchronization for a Bernoulli system with large SLE for different $T_{n}$ values. The parameter are $a=$ 1.1, $\epsilon=0.7, T_{d}=100, N=40$ and $p=1 / 2$ but we restrict the sampled networks such that $\Delta<1 / 10$. This corresponds to a lower bound for $\epsilon^{*}>0.909$. Despite having $\epsilon<\epsilon^{*}$, the system synchronizes in the fast fluctuations regime.

Synchronization by fast network fluctuations has not previously been reported for time-delayed weakly chaotic sytems. This framework is different from the classic fast switching approximation framework, which was developed for linear oscillators with diffusive coupling [26]. Instead, in our setting the couplings are given by the stochastic adjacency matrix and not by the Laplacian 7. Also, the interpretation of the internal time scale $T_{d}$ for time-delayed systems is not so simple as for the case of non-delayed oscillators. In the dynamics of our network we see the interplay of three timescales: the internal time scale (depending on the instantaneous term in Eq. (9)), the large coupling delay, $T_{d} \gg 1$, and the time scale of the fluctuations, $T_{n}$. The necessary time to reach synchronization is given by the SLE, whose magnitude scales inversely with $T_{d}$. Hence, for negative SLE a larger delay time implies a slower decay of the perturbations towards the synchronization manifold. Nevertheless, the observed behavior is qualitatively similar to the fast switching approximation and the stability of synchronization is enhanced as $T_{n} \ll T_{d}$, even for networks with average positive SLE. This result is also reminiscent of the Parrondo games [38, where the alternation of losing strategies can give rise to a winning one: a random sequence of non-synchronizing networks gives rise to synchronization. 


\section{Resynchronization effect in finite size systems}

For network switching values of $T_{n}$ comparable to $T_{d}$ we observe a loss of synchronization both in systems with positive and negative average SLE. For this fluctuation time values the fast switching regime no longer applies. Indeed, the system will spend time both in synchronizing and non-synchronizing networks. When the network reaches a non-synchronizing configuration, it will start to escape the synchronization manifold. Interestingly, in the case of negative average SLE, the system synchronizes again for increasing values of $T_{n} \sim 10 T_{d}$, before loosing synchronization completely in the slow fluctuations regime, as can be seen in Fig. 6 (a). We have investigated this behavior and conclude that the synchronization recovery is a finite-size effect.

We have computed the stationary average synchronization level for the whole range of $T_{n}$ values for Bernoulli systems of different sizes. The results of our simulations are depicted on Fig. 9. As we can see, the synchronization recovery effect for fluctuation values larger than the time delay occurs in relatively small systems. The effect disappears completely when studying systems of size as large as $N=70$. Nevertheless, this synchronizing behavior could have relevance in applications, as experimental systems rarely consist on more than a dozen units. The origin of this effect will be topic of further research.

\section{Synchronization oscillations}

An intriguing feature of the average synchronization level curves in Fig. 6 is the presence of oscillations, which decay with time. The oscillations show a periodicity re-

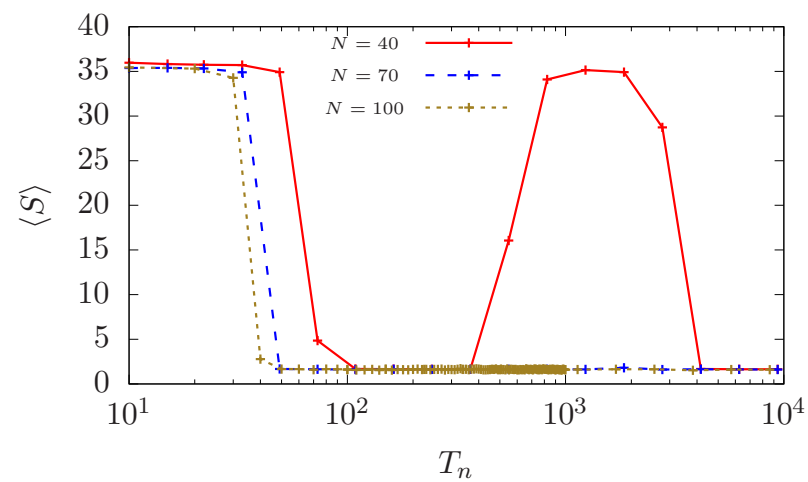

Figure 9: Long term synchronization level Bernoulli map networks of different number of units for a wide range of network switching times. A resynchronizing region appears for intermediate values $T_{n} \gtrsim T_{d}$ for smaller networks, but the effect dissapears for larger systems. Each point corresponds to the average $\mathcal{S}$ over 100 trials after $2 \cdot 10^{6}$ time-steps. In order to verify that we are in a stationary regime, we have checked that the graphic does not qualitatively change when using only $10^{6}$ steps. Parameter values were $a=1.1, T_{d}=100, \epsilon=0.5$ and $p=1 / 2$.
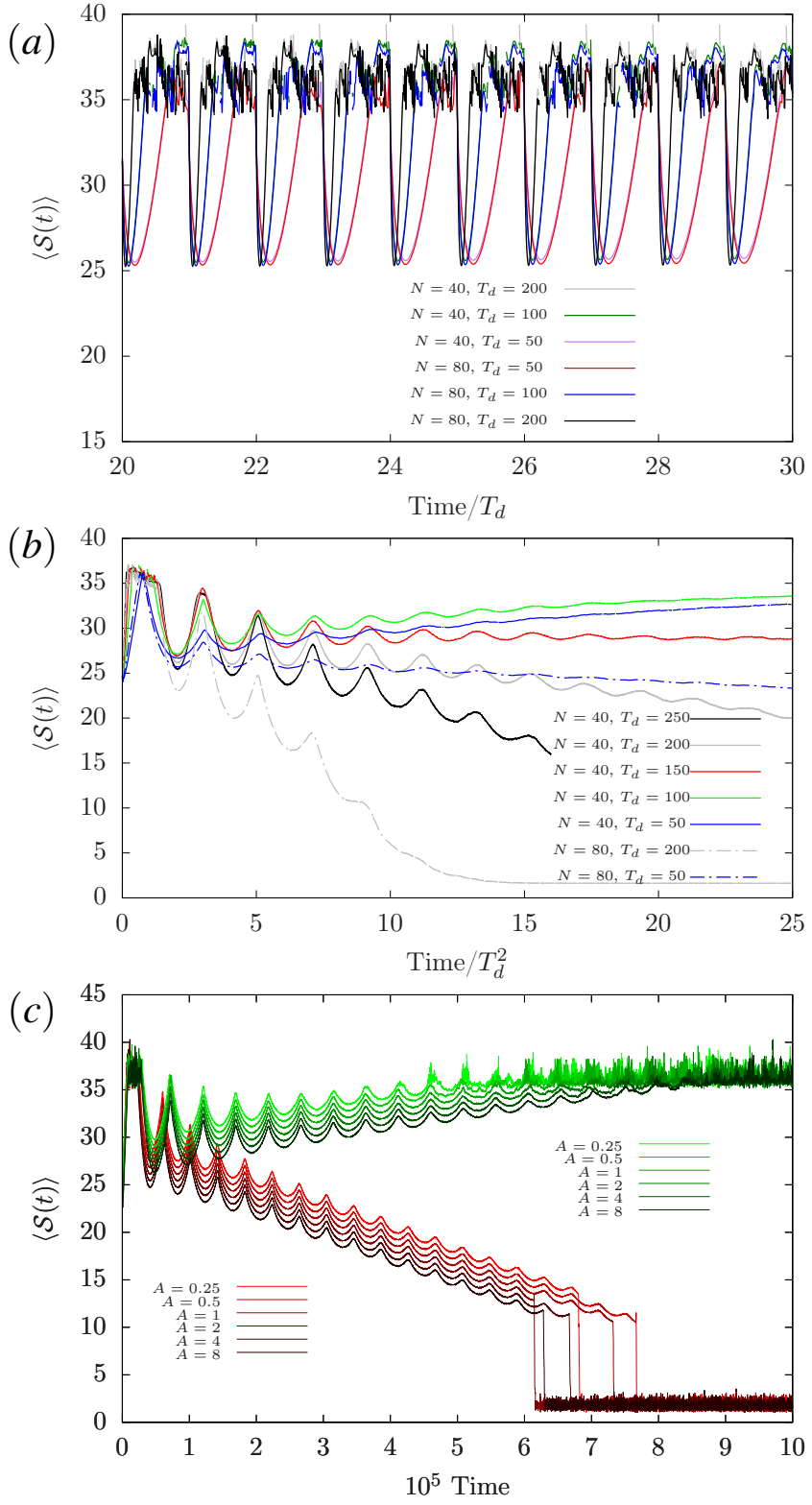

Figure 10: (a) Short-time evolution of the average synchronization level, with time in units of $T_{d}$, for $p=0.5$, for a Bernoulli system with $\epsilon=0.7$ and $T_{n}=1000$, using different values of $T_{d}$ and $N$. (b) Long time evolution, with the short period $T_{d}$ filtered out and time in units of $T_{d}^{2}$. Notice that, in all cases, the oscillations have the same frequency and phase. (c) Average synchronization level for two fixed networks with $N=20, p=0.7$ and $\epsilon=0.83$. The ascending greenish sequence corresponds to a synchronizing instance, while the descending reddish sequence refers to a non-synchronizing one. In both cases, we have averaged over random initial perturbations with amplitude $A \cdot 10^{-10}$. Notice that, in all cases, we obtain oscillations with the same frequency and phase, but different amplitudes. The Bernoulli slope is $a=1.1$ in all cases. 
lated to the time-delay $T_{d}$, and they all have the same phase for different values of the network switching time $T_{n}$. We illustrated this in further detail in Fig. 10 (a), which shows the average synchronization level for a short time-span for all time-steps (not only multiples of 100) using different values of $N$ and $T_{d}$. These oscillations are independent of the network fluctuations, and they appear as well in fixed networks, as shown in Fig. 10 (c).

Besides this primary oscillation we have found a secondary oscillation, whose periodicity scales with the square of the time-delay, $T_{d}^{2}$. In Fig. 10 (b) we have removed the primary period, by showing only times multiple of $T_{d}$, and we represent a longer time span, with the time axis rescaled to $t / T_{d}^{2}$. Again, these fluctuations have the same frequency and phase in all cases.

The oscillations are related to our choice of initial condition: we perturb the system at $t=0$, and this perturbation decays initially. The initial decay is a typical behavior for weakly chaotic systems [28]. After a delay time the perturbation reappears, and this delay echo can be related to the observed periodicity equal to the delay time. For Bernoulli maps the evolution can even be calculated explicitly. We have included the analytic calculations in appendix B

This delay echo is transformed each time-delay interval and its shape gradually changes from an exponential decay to decaying oscillatory motion. In Fig. 11 we show the analytically calculated evolution of a point-like perturbation along a specific direction in a fixed network of Bernoulli elements. While the initial perturbation decays exponentially, the consecutive delay echoes are broader, and reach their maximal amplitude at a later point in time within the delay interval. However, the exact mathematical origin of the observed secondary oscillations remains to be explained.

For general initial conditions in the vicinity of the synchronization manifold, one observes as well one or more frequency components related to the time-delay. We conjecture that they are characteristic for delay systems. We expect however that the phase of the oscillations (and thus the fact that they do not average out over multiple instances) is a result of our choice of initial condition.

\section{CONCLUSIONS AND FURTHER WORK}

The possibility to enhance the stability of a system through fast oscillations or fluctuations is a topic of long tradition, e.g. the Kapitza pendulum [39]. In this work we have explored the effect of topology fluctuations on the stability of synchronization in small-world networks of time-delayed coupled chaotic maps. We have first studied synchronization of static networks sampled from the Newman-Watts small world network ensemble with $N$ nodes and a fraction $p$ of shortcuts. The spectral gap was found to be approximately given by $p / 4$, independently of $N$, and it showed a clear relationship with the synchronization Lyapunov exponent for networks of Bernoulli

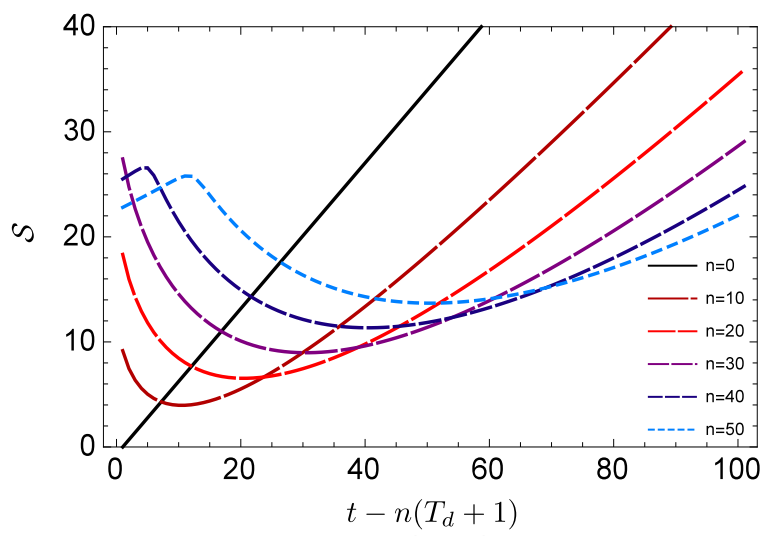

Figure 11: Delay echoes of a point-like perturbation applied at $t=0$ along the transverse direction $v_{2}$, in a network of delay-coupled Bernoulli maps. We show the synchronization level $-\ln \left|v_{2}(t)\right|$ immediately after applying the perturbation, and the tenth, twentieth, thirtieth, fortieth and fiftieth delay echo. We normalized with respect to the initial amplitude. Parameters are $T_{d}=100, a=3 / 2, \epsilon=2 / 3, \gamma_{2}=2 / 5$

and Logistic maps. The Bernoulli map case followed closely the theoretical prediction, while the mapping was more nonlinear for the Logistc maps.

We then studied how the synchronization properties are affected by a time varying coupling topology. We found the stability of the synchronized state to be strongly affected by the interplay between the time-scale of the delayed interactions, $T_{d}$, and that of the network fluctuations, $T_{n}$. For the fast switching regime, $T_{n} \ll T_{d}$, we obtain a strong enhancement of the network's synchronization stability. Even when we restrict our topology fluctuations to only explore those networks which would not be able to synchronize under static conditions, we can obtain almost-sure synchronization under fast enough fluctuations. This result is in qualitative agreement with the fast switching approximation 26. In all cases we observe a severe reduction in the synchronization level for $T_{n} \sim T_{d}$, where the fast fluctuation regime no longer applies. Interestingly, small networks with negative average SLE show a recovery of the synchronization level when increasing $T_{n}>T_{d}$, which is finally lost at higher $T_{n}$ values. This synchronization enhancement is a finite-size effect that is lost when studying networks with a larger number of nodes. Nonetheless, for $T_{n} \gg T_{d}$ the system will nearly always desynchronize. Moreover, we observe oscillations in the synchronization level, when the network is close to the synchronized state. These oscillations have a periodicity related to $T_{d}$ and are typical of the weak chaos regime. For our choice of perturbation, the oscillations can be analytically recovered in a network of Bernoulli maps. We also report a secondary oscillation of periodicity scaling with $T_{d}^{2}$.

We have restricted ourselves to the case of smallworld networks, because they are more amenable to a mean-field approach, but it is relevant to ask whether 
these results also apply to other network ensembles, such as purely random Erdős-Rényi graphs or scale-free networks; as well as to other dynamical systems beyond Bernoulli or Logistic.

\section{Acknowledgments}

We would like to acknowledge W. Kinzel and A. Deaño. This work was partly supported by the Spanish Government through grant FIS-2012-38866-C05-1 (J.R.-L.) and the Alexander von Humboldt Foundation within the Renewed research stay program (E.K.).

\section{Appendix A: Mean-field spectrum and eigengap of the SW networks}

Our ensemble is composed of networks consisting on a directed ring of $N$ nodes, to which we add $N p$ directed shortcuts. Here we compute the spectrum of the networks of this ensemble within the mean-field approximation of 32 , in order to characterize the eigengap. The strategy is as follows: we obtain the ensemble average of each adjacency matrix entry, $\left\langle G_{i j}\right\rangle$, and study the spectrum of the resulting matrix, which will be a circulant matrix,

$$
G^{\mathrm{MF}}=\left[\begin{array}{ccccc}
c_{0} & c_{N-1} & \ldots & c_{2} & c_{1} \\
c_{1} & c_{0} & \ldots & c_{3} & c_{2} \\
\vdots & \vdots & \ddots & \vdots & \vdots \\
c_{N-1} & c_{N-2} & \ldots & c_{1} & c_{0}
\end{array}\right]
$$

The eigenvalues can be computed analytically [27] as

$$
\gamma_{m}^{\mathrm{MF}}=\sum_{k} c_{k} \exp \left[\frac{-2 \pi i m k}{N}\right]
$$

where $m=0, \ldots, N-1$. Before the shortcuts are introduced, $c_{1}=1$ and all other entries $c_{i}=0, i \neq 1$. When the shortcuts are introduced, the subdiagonal elements become $G_{i, i+1}=1 /\left(1+n_{s}\right)$, where $n_{s}$ is the number of shortcuts reaching element $i$. Thus,

$$
c_{1}=\left\langle G_{i, i+1}\right\rangle=\left\langle\frac{1}{1+n_{s}}\right\rangle \text {. }
$$

The random variable $n_{s}$ follows a binomial distribution: each site can be reached by $\approx N$ possible shortcuts, each of them with probability $\approx p / N$. Thus, its probability distribution is given by

$$
P\left(n_{s}\right) \approx\left(\begin{array}{l}
N \\
n_{s}
\end{array}\right)\left(\frac{p}{N}\right)^{n_{s}}\left(1-\frac{p}{N}\right)^{N-n_{s}}
$$

which, in the limit where $p / N \ll 1$ approaches the Poisson distribution

$$
P\left(n_{s}\right) \approx e^{-p} \frac{p^{n_{s}}}{n_{s} !}
$$

For this distribution it is possible to obtain the desired expected value:

$$
\begin{aligned}
c_{1} & =\left\langle\frac{1}{1+n_{s}}\right\rangle \approx \sum_{n_{s}=0}^{\infty} \frac{1}{1+n_{s}} e^{-p} \frac{p^{n_{s}}}{n_{s} !} \\
& =\frac{e^{-p}}{p} \sum_{x=1}^{\infty} \frac{p^{x}}{x !} \\
& =\frac{1-e^{-p}}{p}
\end{aligned}
$$

where we choose $x=n_{s}+1$. The rest of the entries of the circulant matrix are all equal, $c_{i}=\tilde{c}$ for $i \neq 1$, and can be found by normalization:

$$
\tilde{c} \approx \frac{1}{N-1}\left(1-\frac{1-e^{-p}}{p}\right) .
$$

Thus, applying (A2) we have

$$
\gamma_{m}^{\mathrm{MF}}=\tilde{c}+c_{1} \mathrm{e}^{\frac{2 \pi i m}{N}}+\tilde{c} \sum_{k=2}^{N-1} \mathrm{e}^{\frac{2 \pi i k m}{N}}
$$

If $m=0$, we obtain $\gamma_{0}=1$. The last term can be evaluated as a geometric sum or, alternatively, we can realize that if $k$ was extended from 0 to $N-1$, it would yield zero. In both cases, we obtain

$$
\gamma_{m}^{\mathrm{MF}}=\left(c_{1}-\tilde{c}\right) \mathrm{e}^{-\frac{2 \pi i m}{N}} .
$$

Thus, the modulus of all eigenvalues for $m>0$ is equal:

$$
\left|\gamma_{m}^{\mathrm{MF}}\right| \approx c_{1}-\tilde{c}=\frac{1-e^{-p}}{p}-\frac{1}{N-1}\left(1-\frac{1-e^{-p}}{p}\right) .
$$

Neglecting corrections of order $N^{-1}$, the eigenvalue gap is:

$$
\Delta=1-\max \left\{\left|\gamma_{m \neq 0}^{\mathrm{MF}}\right|\right\} \approx 1-\frac{1-e^{-p}}{p}
$$

\section{Appendix B: Analytic explanation of the synchronization oscillations}

We show in this appendix how synchronization oscillations arise generically, as the network decays towards 
or drifts away from the synchronization manifold. We consider a network of Bernoulli maps and the network structure to be fixed.

In this case, we consider a small perturbation around the synchronization manifold, $u_{i}(t)=\mu(t)+\xi_{i}(t)$. The evolution of the network is then given by

$$
\begin{aligned}
& \xi_{i}(t+1)=(1-\epsilon) f^{\prime}(\mu(t)) \xi_{i}(t)+ \\
& \quad \epsilon \sum_{j} G_{i j} f^{\prime}\left(\mu\left(t-T_{d}\right)\right) \xi_{j}\left(t-T_{d}\right) .
\end{aligned}
$$

After decomposition along the eigenvectors $\left\{v_{k}\right\}$ of $G$, we can rewrite Eq. B1 as

$$
\begin{aligned}
v_{k}(t+1)=(1-\epsilon) f^{\prime}(\mu(t)) v_{k}(t)+ \\
\epsilon \gamma_{k} f^{\prime}\left(\mu\left(t-T_{d}\right)\right) v_{k}\left(t-T_{d}\right),
\end{aligned}
$$

where $\gamma_{k}$ denotes the eigenvalue of $G$ along the eigenvector $v_{k}$. For Bernoulli maps, the derivative along the chaotic trajectory is constant, $f^{\prime}(u(t))=a$, and we can simplify Eq. (B2) as

$$
v_{k}(t+1)=(1-\epsilon) a v_{k}(t)+\epsilon \gamma_{k} a v_{k}\left(t-T_{d}\right) .
$$

The exponential decay of a perturbation is slowest (or the growth is fastest) along the direction with the smallest eigenvalue gap $1-\left|\gamma_{2}\right|$, we thus only consider the direction $v_{2}(t)$. To find the evolution along a direction $v_{k}$, one can simply replace $\gamma_{2}$ by $\gamma_{k}$ in the calculations. In the simulations we applied a perturbation $\boldsymbol{\xi}(t)$ only at $t=0$, with a randomized magnitude over the network nodes. For simplicity, we will assume this magnitude along the direction $v_{2}(0)=1$, while $v_{2}(t<0)=0$. We can then solve Eq. B3) directly, and we find for the first delay interval, $0 \leq t<T_{d}$,

$$
v_{2}(t+1)=(1-\epsilon) a v_{2}(t),
$$

which is solved by

$$
v_{2}(t)=((1-\epsilon) a)^{t} \text { for } 0<t \leq T_{d} .
$$

The perturbation initially evolves with a rate given by $\ln |(1-\epsilon) a|$, which corresponds to the instantaneous Lyapunov exponent 7,28 . We only consider networks in the weakly chaotic regime, meaning that the instantaneous Lyapunov exponent is negative and that the perturbation initially decays.

Using Eq. (B5) as initial function for the next delay interval $T_{d} \leq t<2 T_{d}+1$, this leads to an equation of motion

$$
v_{2}(t+1)=(1-\epsilon) a v_{2}(t)+\epsilon a \gamma_{2}((1-\epsilon) a)^{t} .
$$

Imposing continuity, $v_{2}\left(T_{d}\right)=((1-\epsilon) a)^{T_{d}}$, this difference equation is solved by

$$
v_{2}(t)=((1-\epsilon) a)^{t}+\left(t-T_{d}\right) \epsilon a \gamma_{2}((1-\epsilon) a)^{t-T_{d}-1}
$$

for $T_{d}<t \leq 2 T_{d}+1$. Hence, the initial perturbation reappears after a time $T_{d}+1$, but the delay echo is broadened. In general, we find for $n\left(T_{d}+1\right) \leq t<(n+1)\left(T_{d}+1\right)$

$v_{2}(t)=\sum_{k=0}^{n} \frac{1}{k !}((1-\epsilon) a)^{t-k T_{d}-k}\left(a \epsilon \gamma_{2}\right)^{k} \prod_{l=0}^{k-1}\left(t-k T_{d}-l\right)$.

We find additional delay echoes appearing at multiples of $T_{d}+1$, each one broader than the previous. After several cycles the resulting motion indeed resembles an oscillation, with a periodicity approximated as

$$
T \approx T_{d}+1 / 2-1 / \ln |a(1-\epsilon)|
$$

in the limit of large delay $T_{d} \rightarrow \infty$. This is illustrated in Fig. 11. We remark here that in general, $\gamma_{2}$ is a complex number, which could lead to additional oscillations with a periodicity related to the delay time and the phase of $\gamma_{2}$.

\section{References}

[1] S. Boccaletti, J. Kurths, G. Osipov, D. Valladares, and C. Zhou, Physics Reports 366, 1 (2002).

[2] L. M. Pecora and T. L. Carroll, Phys. Rev. Lett. 80, 2109 (1998).

[3] V. Flunkert, S. Yanchuk, T. Dahms, and E. Schöll, Phys. Rev. Lett. 105, 254101 (2010).

[4] F. M. Atay, J. Jost, and A. Wende, Phys. Rev. Lett. 92, 144101 (2004)

[5] T. Heil, I. Fischer, W. Elsäßer, J. Mulet, and C. R. Mirasso, Phys. Rev. Lett. 86, 795 (2001).

[6] I. Fischer, R. Vicente, J. M. Buldu, M. Peil, C. R. Mirasso, M. C. Torrent, and J. Garcia-Ojalvo, Phys. Rev.
Lett. 97, 123902 (2006).

[7] S. Heiligenthal, T. Dahms, S. Yanchuk, T. Jüngling, V. Flunkert, I. Kanter, E. Schöll, and W. Kinzel, Phys. Rev. Lett. 107, 234102 (2011).

[8] M. Nixon, M. Friedman, E. Ronen, A. A. Friesem, N. Davidson, and I. Kanter, Phys. Rev. Lett. 108, 214101 (2012).

[9] A. Argyris, D. Syvridis, L. Larger, V. Annovazzi-Lodi, P. Colet, I. Fischer, J. García-Ojalvo, C. R. Mirasso, L. Pesquera, and K. A. Shore, Nature 438, 343 (2005).

[10] I. Kanter, E. Kopelowitz, and W. Kinzel, Phys. Rev. Lett. 101, 084102 (2008).

[11] G. Buzsaki, Rhythms of the brain (Oxford University Press, 2006). 
[12] I. Kanter, E. Kopelowitz, R. Vardi, M. Zigzag, W. Kinzel, M. Abeles, and D. Cohen, EPL (Europhysics Letters) 93, 66001 (2011).

[13] I. Kanter, M. Zigzag, A. Englert, F. Geissler, and W. Kinzel, EPL (Europhysics Letters) 93, 60003 (2011).

[14] M. J. Martin, O. D'Huys, L. Lauerbach, E. Korutcheva, and W. Kinzel, Phys. Rev. E 93, 022206 (2016).

[15] J. Feng, V. K. Jirsa, and M. Ding, Chaos 16, 015109 (2006).

[16] P. Holme, The European Physical Journal B 88, 1 (2015).

[17] D. V. Buonomano and M. M. Merzenich, Annual Review of Neuroscience 21, 149 (1998), pMID: 9530495.

[18] I. V. Belykh, V. N. Belykh, and M. Hasler, Physica D: Nonlinear Phenomena 195, 188 (2004).

[19] F. Peruani, E. M. Nicola, and L. G. Morelli, New Journal of Physics 12, 093029 (2010).

[20] N. Fujiwara, J. Kurths, and A. Díaz-Guilera, Phys. Rev. E 83, 025101 (2011).

[21] M. Frasca, A. Buscarino, A. Rizzo, L. Fortuna, and S. Boccaletti, Phys. Rev. Lett. 100, 044102 (2008).

[22] N. Fujiwara, J. Kurths, and A. Díaz-Guilera, Chaos 26, 094824 (2016).

[23] K. Uriu, S. Ares, A. C. Oates, and L. G. Morelli, Phys. Rev. E 87, 032911 (2013).

[24] K. Uriu and L. G. Morelli, Biophys J 107, 514 (2014).

[25] M. Nag and S. Poria, Chaos, Solitons \& Fractals 91, 9 (2016).

[26] D. J. Stilwell, E. M. Bollt, and D. G. Roberson, SIAM Journal on Applied Dynamical Systems 5, 140 (2006)

[27] G. H. Golub and C. F. Van Loan, Matrix Computations (Johns Hopkins University Press, 1996).
[28] O. D'Huys, S. Zeeb, T. Jüngling, S. Yanchuk, and W. Kinzel, EPL (Europhysics Letters) 103, 10013, (2013).

[29] T. Jüngling, O. D'Huys, and W. Kinzel, Phys. Rev. E 91, 062918 (2015).

[30] M. Barahona and L. M. Pecora, Phys. Rev. Lett. 89, 054101 (2002).

[31] Y. Aviad, I. Reidler, M. Zigzag, M. Rosenbluh, and I. Kanter, Opt. Express 20, 4352 (2012).

[32] C. Grabow, S. Grosskinsky, J. Kurths, and M. Timme, Phys. Rev. E 91, 052815 (2015).

[33] M. Newman and D. Watts, Physics Letters A 263, 341 (1999).

[34] D. J. Watts and S. H. Strogatz, Nature 393, 409 (1998).

[35] R. Kühn, Journal of Physics A: Mathematical and Theoretical 41, 295002 (2008).

[36] J. Billen, M. Wilson, A. Baljon, and A. Rabinovitch, Phys. Rev. E 80, 046116 (2009).

[37] A. Knoblauch, F. Hauser, M.-O. Gewaltig, E. Körner, and G. Palm, Frontiers in computational neuroscience 6, 55 (2012).

[38] G. P. Harmer and D. Abbott, Nature 402, 864 (1999).

[39] L. Landau and E. Lifshitz, Mechanics (Pergamon Press, 1960).

[40] S. N. Elaydi, Discrete chaos: with applications in science and engineering (CRC Press, 2007).

[41] The Lyapunov exponent of an isolated Bernoulli map is $\lambda_{\mathrm{B}}=\ln a \approx 0.09531$. The Lyapunov exponent of the Logistic map, $\lambda_{\mathrm{L}}$, must be obtained computationally, but a value of $r=3.577$ gives $\lambda_{\mathrm{B}} \approx \lambda_{\mathrm{L}}, 40$. 